\title{
Efeito da dexametasona e melatonina exógenas sobre parâmetros sanguíneos, progesterona, carboidratos totais e histomorfometria de órgãos em ratas prenhes
}

\author{
[The effect of dexamethasone and exogenous melatonin on blood parameters, progesterone, total \\ carbohydrates and histomorphometry of organs in pregnant rats]
}

\author{
S.B. Silva, A.F. Soares, V. Wanderley-Teixeira*, A.A.C. Teixeira, \\ P.E.A. Vilaça-Júnior, M.T.J.A. Castanho
}

Universidade Federal Rural de Pernambuco - UFRPE - Recife, PE

\begin{abstract}
RESUMO
A dexametasona é utilizada em casos de gestação com risco de prematuridade; porém, doses suprafisiológicas podem afetar a embriogênese. A melatonina tem demonstrado prevenir efeitos deletérios dos glicocorticoides. Assim, avaliamos a influência da melatonina sobre efeitos da dexametasona em ratas prenhes através dos seguintes parâmetros: 1. Hemograma e perfil glicídico; 2. Níveis de progesterona; e 3. Histomorfometria e histoquímica. Foram utilizadas 20 ratas divididas nos grupos: I - ratas prenhes que receberam placebo (Controle); II - ratas prenhes tratadas com dexametasona $(0,8 \mathrm{mg} / \mathrm{kg})$; III - ratas prenhes tratadas com melatonina $(0,5 \mathrm{mg} / \mathrm{kg})$; IV - ratas prenhes tratadas com dexametasona e melatonina. Todos os tratamentos foram iniciados 10 dias após confirmação do acasalamento até o final da prenhez. O sangue foi coletado no $7^{\circ}$, $14^{\circ}$ e $21^{\circ}$ dia de prenhez. As dosagens de carboidratos e progesterona foram realizadas pelo método antrona e ELISA, respectivamente. O fígado, rins e adrenais foram analisados histoquímica e morfometricamente. No $7^{\circ}$ dia de prenhez, não houve alteração significativa nos parâmetros analisados. Porém, no $14^{\circ}$ dia de prenhez, houve aumento significativo do volume de hematócrito, redução do número total de hemácias e leucócitos, neutrofilia, linfopenia, eosinopenia e redução do diâmetro das hemácias nas matrizes tratadas com dexametasona. Esses efeitos permaneceram no $21^{\circ}$ dia de prenhez, exceto para o hematócrito, o qual reduziu. Verificou-se ainda redução significativa dos níveis de glicose $\left(21^{\circ}\right.$ dia de prenhez) e progesterona $\left(14^{\circ}\right.$ e $21^{\circ}$ dia de prenhez). Não houve alteração nos parâmetros morfométricos e histoquímico no fígado, rins e adrenais. A dexametasona na dosagem de $0,8 \mathrm{mg} / \mathrm{kg}$, administrada a partir do terço médio da prenhez, produz alterações hematológicas, bioquímicas e hormonais em ratas, sendo prevenidas pela melatonina; porém não afeta o fígado, rins e adrenais quanto aos parâmetros morfométricos e histoquímicos.
\end{abstract}

Palavras-chave: ratas, melatonina, glicocorticoide, hemograma, gestação

\begin{abstract}
Dexamethasone is used in cases of pregnancy with prematurity risk. However, it may affect the embryogenesis when used in supraphysiological doses. Melatonin has been shown to prevent the deleterious effects of glucocorticoids. Therefore, the influence of melatonin on the effects of dexamethasone on pregnant rats was evaluated through the following parameters: 1. Hemogram and glucose profile; 2. Progesterone levels; 3. Histomorphometry and histochemistry analyses. Twenty female rats were divided into the following groups: Irats that received placebo (Control); II -rats treated with dexamethasone $(0.8 \mathrm{mg} / \mathrm{kg})$; III - rats treated with melatonin $(0.5 \mathrm{mg} / \mathrm{kg}) ; \mathrm{IV}$ - rats treated with dexamethasone and melatonin. All treatments started 10 days after confirmation of mating and lasted until the end of the pregnancy. Blood samples were collected on the $7^{\text {th }}, 14^{\text {th }}$, and $21^{\text {st }}$ day. Carbohydrate and progesterone levels were determined with the antrona and ELISA method, respectively. The liver, kidneys, and adrenal glands were analyzed morphometrically and histochemically. On the $7^{\text {th }}$ day of pregnancy there were no significant changes in the parameters analyzed. However, at 14 days of pregnancy there was a significant increase of hematocrit, reducing the total number of erythrocytes and leukocytes, neutrophilia, lymphopenia, eosinopenia and reduced diameter of red blood cells in rats treated with
\end{abstract}

Recebido em 11 de abril de 2012

Aceito em 5 de setembro de 2013

*Autor para correspondência (corresponding author)

E-mail: valeria@dmfa.ufrpe.br 
dexamethasone. These effects remained on the $21^{\text {st }}$ of day of pregnancy, except for the hematocrit, which was reduced. There was also a significant reduction in glucose levels $\left(21^{\text {st }}\right.$ day) and progesterone $\left(14^{\text {th }}\right.$ and $21^{\text {st }}$ days). There was no change in the histochemical and morphometric parameters in the liver, kidneys and adrenals. Dexamethasone at a dosage of $0.8 \mathrm{mg} / \mathrm{kg}$ administered from the middle third of pregnancy produces hematological, biochemical and hormonal changes in rats, being prevented by melatonin, but does not affect the liver, kidneys and adrenal glands regarding morphometric and histochemical parameters.

Keywords: rats, melatonin, glucocorticoid, hemogram, pregnancy

\section{INTRODUÇÃO}

A dexametasona é um glicocorticoide sintético com ação anti-inflamatória e imunossupressora (Bavaresco et al., 2005). Esse glicocorticoide tem sido utilizado em gestações com risco de prematuridade por acelerar o desenvolvimento de tecidos-alvos no feto, possibilitando um aumento na probabilidade de sobrevivência do prematuro (Locatelli et al., 2010). Doses suprafisiológicas de dexametasona, no entanto, podem propiciar complicações, como osteoporose, osteonecrose, insuficiência da glândula suparrenal, imunodepressão, hiperglicemia, entre outras (Rhodus et al., 2006).

O tratamento com dexametasona pré-natal pode afetar ainda o desenvolvimento e função da placenta, comprometendo a embriogênese (Lee et al., 2012). Alguns órgãos, como o pâncreas, o fígado e os rins, podem apresentar alterações morfofuncionais atribuídas à corticoterapia (Bruder et al., 2006). A dexametasona ainda exerce influência sobre a distribuição de células sanguíneas através da inibição da expressão de citocinas (Remmelts et al., 2012), redução drástica do número de linfócitos $\mathrm{T}$, elevação acentuada de neutrófilos, além de uma redução acentuada dos linfócitos B, células NK (natural killer) e monócitos (Dhabhar et al., 1996).

Segundo Aoyama et al. (1986), a administração de melatonina, uma indolamina produzida pela pineal, preveniu em ratas jovens os efeitos deletérios, tais como diminuição do ganho de peso corporal, atrofia do timo e das adrenais, glicosúria, elevação do nível de açúcar no sangue, triglicerídeos e colesterol total, causados por três diferentes glicocorticoides: dexametasona, prednisolona e hidrocortisona.

Os estudos que tratam das alterações de alguns órgãos, como fígado, rins e adrenais, relacionadas ao uso de corticoides, apontam para a melatonina como um fator capaz de reduzir os danos causados por esses efeitos, uma vez que a melatonina exerce influência sobre os corticoides naturalmente produzidos pelas adrenais e sobre hormônios esteroides. Dessa forma, o presente estudo teve como objetivo relacionar a influência do uso de melatonina sobre os efeitos sistêmicos da dexametasona por meio dos seguintes parâmetros: 1. Hemograma e perfil glicídico; 2 . Níveis de progesterona; e 3. Histomorfometria do fígado, rins, adrenais e hemácias de ratas prenhes.

\section{MATERIAL E MÉTODOS}

Foram utilizadas 20 ratas albinas (Rattus norvegicus albinus) com 90 dias de idade, virgens, pesando $200 \pm 30 \mathrm{~g}$, da linhagem Wistar, procedentes do Biotério do Departamento de Morfologia e Fisiologia Animal, da Universidade Federal Rural de Pernambuco - UFRPE. As fêmeas foram confinadas em gaiolas e mantidas com alimentação e água "add libitum", a uma temperatura de $22^{\circ} \mathrm{C}$ e iluminação artificial com fotoperíodo de 12 horas luz/12 horas escuro. O protocolo experimental foi aprovado pela Comissão de Ética institucional, de $\mathrm{n}^{\circ}$. 23082.017195/2010. As fêmeas foram distribuídas em quatro grupos recebendo os seguintes tratamentos 10 dias após a confirmação da prenhez: placebo (I); dexametasona (II); melatonina (III); dexametasona e melatonina (IV). Foi utilizada a formulação comercial Decadron $^{\circledR}$ (fosfato dissódico de dexametasona), administrada i.p., na dose de $0,8 \mathrm{mg} / \mathrm{kg} / \mathrm{animal}$ (Vilaça Júnior et al., 2012), com redução progressiva da dose em $10 \%$ até obtenção da dose zero no último dia de administração. A melatonina (Sigma Chemical Co., St. Louis, Mo., USA) foi administrada na dosagem de $0,5 \mathrm{mg} / \mathrm{kg}$ i.p. (Subramanian et al., 2007). Como placebo administraram-se solução salina e etanol (3:1) em $\mathrm{pH}$ fisiológico. Todos os tratamentos foram realizados entre $17 \mathrm{~h}$ e $18 \mathrm{~h}$. 
O sangue foi coletado, com seringas contendo EDTA, por punção da veia caudal lateral no $7^{\circ}$, $14^{\circ}$ e $21^{\circ}$ dia de prenhez, centrifugado, e o plasma foi acondicionado a $-20^{\circ} \mathrm{C}$, para análises posteriores. Após a última coleta, as fêmeas foram anestesiadas com xilazina $(20 \mathrm{mg} / \mathrm{kg})$ e quetamina $(10 \mathrm{mg} / \mathrm{kg})$, por via i.p., para remoção total do fígado, rins e adrenais. Para o hematócrito, alíquotas de sangue total foram coletadas em capilares heparinizados e centrifugadas por cinco minutos a 7000rpm. Na contagem total de hemácias, as alíquotas foram diluídas em solução de Hayem na proporção de 1: 400 e contadas em câmara de Neubauer. Os resultados foram submetidos à fórmula $\mathrm{Hm}$ x 10 x 400 x $5=\mathrm{Hm} / \mu \mathrm{L}$, em que: Hm é o número total de hemácias contadas, 10 é o fator de conservação para o volume de $1 \mathrm{~mm}^{3}$ (profundidade da câmara de Neubauer), 400 é o fator da conservação da diluição utilizada, e 5 é o fator de conservação para área de $1 \mathrm{~mm}^{2}$ ( 5 de 25 quadrados médios), sendo o resultado expresso como o número de hemácias $/ \mu \mathrm{L}$. Para a contagem total de leucócitos as alíquotas foram diluídas em solução de Turk na proporção de 1:20. Os resultados foram submetidos à fórmula: Lc $\times 20 \times 10 / 4=\mathrm{Lc} / \mu \mathrm{L}$, em que: Lc é o número total de leucócitos contados, 10 é o fator de conservação para o volume de $1 \mathrm{~mm}^{3}$ (profundidade da lâmina da câmara de Neubauer), 20 é o fator de conservação da diluição utilizada e 4 é o fator de conservação para a área de $1 \mathrm{~mm}^{2}$ (número de quadrados), sendo o resultado expresso como o número de leucócitos $/ \mu \mathrm{L}$. Para contagem diferencial de leucócitos, foram confeccionados esfregaços de sangue total corados pelo método de panótico rápido. Para análise de carboidratos totais, seguiu-se o protocolo descrito por Laurentini e Edwards (2003), para leitura em microplaca (Anthos 2010 Microplate Absorbance Reader, Biochrom Ltd). Os níveis do hormônio progesterona foram dosados utilizando-se o método Enzyme Linked Immunosorbet Assay (ELISA), através de kits comerciais. Todas as análises hematológicas, dosagens de carboidratos e hormonal foram realizadas em triplicata. Os órgãos coletados foram fixados em formol $10 \%$ tamponado por 24 horas e processados para inclusão em parafina. Os cortes foram submetidos à técnica de coloração pela hematoxilina-eosina (HE). Para histoquímica do fígado e adrenais, empregaram-se os corantes ácido periódico de Schiff (PAS) e impregnação argêntica, respectivamente. Para o estudo morfométrico do fígado utilizou-se a metodologia descrita por Engelman et al. (2001), em cinco lâminas por grupo para análise do percentual do parênquima lobular e não lobular. Para os rins, foram utilizadas cinco lâminas de cada grupo, e analisados 10 glomérulos/lâmina que demonstraram, num único corte, os polos vascular e urinário. A captura da imagem foi efetuada por meio de câmera de Vídeo Sony ${ }^{\circledR}$, acoplada ao microscópio Olympus ${ }^{\circledR}$ Bx50. Realizou-se a morfometria através de aplicativo Morfometria de Linhas, calibrado em micrômetros, associado ao programa Optimas ${ }^{\circledR}$ 6.2 para Windows. Foi utilizada a mesma metodologia para a mensuração da cápsula de Bowman (Akaoka et al., 1994), e da cortical e medular das adrenais. Para morfometria das hemácias no período de 14 e 21 dias de gestação, foram utilizadas 15 lâminas/grupo/período, sendo mensuradas 100 células/lâmina por meio de régua milimetrada. Os dados dos parâmetros hematológicos, carboidratos totais, níveis hormonais, bem como os dados morfométricos das hemácias, adrenais, fígado e rins, foram submetidos ao teste não paramétrico de KruskalWallis, em que as médias obtidas foram comparadas pelo teste de Wilcoxon MannWhitney $(\mathrm{p}<0,05)$.

\section{RESULTADOS}

Nas Tabelas 1, 2 e 3, estão sumarizadas as médias e desvio padrão do hematócrito, número total de hemácias, número total de leucócitos e contagem diferencial dos leucócitos, das matrizes, no $7^{\circ}, 14^{\circ}$ e $21^{\circ}$ dias de gestação, respectivamente. No $7^{\circ}$ dia de gestação, não houve alteração significativa nos parâmetros analisados nos grupos experimentais (Tab. 1). No entanto, aos 14 dias de gestação, houve aumento significativo do volume de hematócrito e redução do número total de hemácias e leucócitos nas matrizes tratadas com dexametasona. A contagem diferencial dos leucócitos revelou uma significativa neutrofilia, linfopenia e eosinopenia nas matrizes tratadas com dexametasona, em relação aos outros grupos experimentais (Tab. 2). No terço final da gestação, as fêmeas do grupo tratado com dexametasona apresentaram redução dos valores de hematócrito, redução do número total de hemácias e leucócitos, além de neutrofilia, linfopenia e eosinopenia (Tab. 3). Após ter constatado variação significativa no 
hematócrito e na contagem total de hemácias e leucócitos nas ratas tratadas apenas com dexametasona nos intervalos de 14 e 21 dias, repetiu-se o experimento nesse grupo e no controle para análises aos 14, 16, 18 e 20 dias de prenhez. Essas análises revelaram que as hemácias e os leucócitos diminuíram progressivamente ao longo dos intervalos analisados, bem como os linfócitos e eosinófilos, porém os neutrófilos aumentaram progressivamente (Tabs. 4, 5, 6 e 7). De acordo com análise estatística, verificou-se que, no grupo tratado com dexametasona, em relação ao grupo controle, o valor do hematócrito apresentou-se elevado aos 14 dias, normalizando-se aos 16 e 18 dias, porém reduzindo significativamente no $20^{\circ}$ dia. Com o intuito de verificar uma possível anisocitose, realizou-se medição do diâmetro das hemácias de todos os grupos experimentais, sendo observadas hemácias com diâmetro significativamente menor nos animais tratados com dexametasona (Tab. 8).

Tabela 1. ${ }^{1}$ Médias de hematócrito, contagem total e diferencial das células sanguíneas das ratas com 7 dias de gestação

\begin{tabular}{lccccc}
\multicolumn{1}{c}{ Grupos } & I & II & III & IV & P \\
\hline Ht $(\%)$ & $52,73 \pm 2,44 \mathrm{a}$ & $49,66 \pm 3,53 \mathrm{a}$ & $51,00 \pm 1,90 \mathrm{a}$ & $48,80 \pm 2,32 \mathrm{a}$ & 0,1012 \\
CTH $\left(10^{6} / \mu \mathrm{L}\right)$ & $4,76 \pm 1,24^{\mathrm{a}}$ & $4,44 \pm 1,49 \mathrm{a}$ & $4,71 \pm 1,24 \mathrm{a}$ & $4,88 \pm 1,90 \mathrm{a}$ & 0,1930 \\
CTL $\left(10^{3} / \mu \mathrm{L}\right)$ & $2,63 \pm 0,99^{\mathrm{a}}$ & $2,68 \pm 0,89 \mathrm{a}$ & $2,65 \pm 0,53 \mathrm{a}$ & $2,70 \pm 0,87 \mathrm{a}$ & 0,2121 \\
Neutrófilos $(\%)$ & $13,60 \pm 2,61 \mathrm{a}$ & $14,13 \pm 1,57 \mathrm{a}$ & $12,46 \pm 1,80 \mathrm{a}$ & $14,63 \pm 2,89 \mathrm{a}$ & 0,4261 \\
Linfócitos $(\%)$ & $86,06 \pm 4,68 \mathrm{a}$ & $87,33 \pm 5,03 \mathrm{a}$ & $84,13 \pm 3,33 \mathrm{a}$ & $82,54 \pm 2,23 \mathrm{a}$ & 0,0838 \\
Eosinófilos $(\%)$ & $1,45 \pm 0,26^{\mathrm{a}}$ & $1,49 \pm 0,29 \mathrm{a}$ & $1,20 \pm 0,18 \mathrm{a}$ & $1,34 \pm 0,21 \mathrm{a}$ & 0,5102 \\
Monócitos $(\%)$ & $0,53 \pm 0,44^{\mathrm{a}}$ & $0,40 \pm 0,36 \mathrm{a}$ & $0,58 \pm 0,31 \mathrm{a}$ & $0,49 \pm 0,32 \mathrm{a}$ & 0,7581 \\
Basófilos $(\%)$ & $0,13 \pm 0,29^{\mathrm{a}}$ & $0,06 \pm 0,15 \mathrm{a}$ & $0,20 \pm 0,13 \mathrm{a}$ & $0,18 \pm 0,11 \mathrm{a}$ & 0,7359 \\
\hline
\end{tabular}

${ }^{1}$ Médias seguidas pela mesma letra não diferem significativamente entre si pelo teste de Wilcoxon-Mann-Whitney $(\mathrm{P}<0,05)$.

Tabela 2. ${ }^{1}$ Médias de hematócrito, contagem total e diferencial das células sanguíneas das ratas com 14 dias de gestação

\begin{tabular}{lccccc}
\multicolumn{1}{c}{ Grupos } & I & II & III & IV & P \\
\hline Ht $(\%)$ & $42,93 \pm 4,21 \mathrm{a}$ & $52,60 \pm 3,83 \mathrm{~b}$ & $40,73 \pm 1,65 \mathrm{a}$ & $38,20 \pm 2,90 \mathrm{a}$ & 0,0005 \\
$\mathrm{CTH}\left(10^{6} / \mu \mathrm{L}\right)$ & $4,66 \pm 1,56 \mathrm{a}$ & $3,86 \pm 1,46 \mathrm{~b}$ & $4,86 \pm 1,13 \mathrm{a}$ & $4,72 \pm 1,76 \mathrm{a}$ & 0,0002 \\
$\mathrm{CTL}\left(10^{3} / \mu \mathrm{L}\right)$ & $2,64 \pm 1,65 \mathrm{a}$ & $1,81 \pm 1,54 \mathrm{~b}$ & $2,62 \pm 1,27 \mathrm{a}$ & $2,49 \pm 0,92 \mathrm{a}$ & 0,0014 \\
Neutrófilos $(\%)$ & $31,73 \pm 3,76 \mathrm{a}$ & $40,93 \pm 2,11 \mathrm{~b}$ & $33,80 \pm 1,12 \mathrm{a}$ & $29,66 \pm 3,08 \mathrm{a}$ & 0,0101 \\
Linfócitos $(\%)$ & $86,00 \pm 4,04 \mathrm{a}$ & $72,99 \pm 5,56 \mathrm{~b}$ & $88,26 \pm 6,82 \mathrm{a}$ & $84,38 \pm 4,44 \mathrm{a}$ & 0,0270 \\
Eosinófilos $(\%)$ & $1,66 \pm 0,13 \mathrm{a}$ & $0,63 \pm 0,14 \mathrm{~b}$ & $1,39 \pm 0,11 \mathrm{a}$ & $1,42 \pm 0,12 \mathrm{a}$ & 0,0441 \\
Monócitos $(\%)$ & $0,33 \pm 0,23 \mathrm{a}$ & $0,26 \pm 0,27 \mathrm{a}$ & $0,47 \pm 0,14 \mathrm{a}$ & $0,56 \pm 0,12 \mathrm{a}$ & 0,6010 \\
Basófilos $(\%)$ & $0,34 \pm 0,18 \mathrm{a}$ & $0,26 \pm 0,12 \mathrm{a}$ & $0,40 \pm 0,16 \mathrm{a}$ & $0,29 \pm 0,17 \mathrm{a}$ & 0,8580 \\
\hline
\end{tabular}

${ }^{1}$ Médias seguidas pela mesma letra não diferem significativamente entre si pelo teste de Wilcoxon-Mann-Whitney $(\mathrm{P}<0,05)$.

Tabela 3. ${ }^{1}$ Médias do hematócrito, contagem total e diferencial das células sanguíneas das ratas com 21 dias de gestação

\begin{tabular}{lccccc}
\multicolumn{1}{c}{ Grupos } & I & II & III & IV & P \\
\hline Ht $(\%)$ & $43,13 \pm 5,22 \mathrm{a}$ & $28,66 \pm 4,61 \mathrm{~b}$ & $45,33 \pm 3,71 \mathrm{a}$ & $39,00 \pm 5,73 \mathrm{a}$ & 0,0055 \\
$\mathrm{CTH}\left(10^{6} / \mu \mathrm{L}\right)$ & $4,07 \pm 1,40 \mathrm{a}$ & $2,96 \pm 1,84 \mathrm{~b}$ & $3,94 \pm 1,17 \mathrm{a}$ & $4,18 \pm 1,69 \mathrm{a}$ & 0,0160 \\
$\mathrm{CTL}\left(10^{3} / \mu \mathrm{L}\right)$ & $2,68 \pm 0,97 \mathrm{a}$ & $1,80 \pm 0,57 \mathrm{~b}$ & $2,57 \pm 0,91 \mathrm{a}$ & $2,66 \pm 0,43 \mathrm{a}$ & 0,0001 \\
Neutrófilos $(\%)$ & $46,60 \pm 3,62 \mathrm{a}$ & $55,06 \pm 3,83 \mathrm{~b}$ & $44,79 \pm 3,44 \mathrm{a}$ & $43,78 \pm 2,65 \mathrm{a}$ & 0,0370 \\
Linfócitos $(\%)$ & $89,46 \pm 6,14 \mathrm{a}$ & $77,06 \pm 5,30 \mathrm{~b}$ & $84,86 \pm 5,44 \mathrm{a}$ & $86,94 \pm 6,76 \mathrm{a}$ & 0,0196 \\
Eosinófilos $(\%)$ & $1,76 \pm 0,18 \mathrm{a}$ & $1,26 \pm 0,16 \mathrm{~b}$ & $1,65 \pm 0,14 \mathrm{a}$ & $1,77 \pm 0,11 \mathrm{a}$ & 0,0368 \\
Monócitos $(\%)$ & $0,66 \pm 0,10 \mathrm{a}$ & $0,46 \pm 0,19 \mathrm{a}$ & $0,60 \pm 0,22 \mathrm{a}$ & $0,58 \pm 0,18 \mathrm{a}$ & 0,1115 \\
Basófilos $(\%)$ & $0,27 \pm 0,15 \mathrm{a}$ & $0,26 \pm 0,12 \mathrm{a}$ & $0,40 \pm 0,16 \mathrm{a}$ & $0,38 \pm 0,13 \mathrm{a}$ & 0,8322 \\
\hline
\end{tabular}

${ }^{\mathrm{T}}$ Médias seguidas pela mesma letra não diferem significativamente entre si pelo teste de Wilcoxon-Mann-Whitney $(\mathrm{P}<0,05)$. 
Efeito da dexametasona...

Tabela 4. ${ }^{1}$ Médias de hematócrito, contagem total e diferencial das células sanguíneas das ratas com 14 dias de gestação

\begin{tabular}{lccc}
\hline & I & II & P \\
\hline Ht $(\%)$ & $40,60 \pm 2,96 \mathrm{a}$ & $51,00 \pm 3,53 \mathrm{~b}$ & 0,0079 \\
$\mathrm{CTH}\left(10^{6} / \mu \mathrm{L}\right)$ & $4,80 \pm 1,60 \mathrm{a}$ & $3,81 \pm 1,72 \mathrm{~b}$ & 0,0195 \\
$\mathrm{CTL}\left(10^{3} / \mu \mathrm{L}\right)$ & $2,51 \pm 1,29 \mathrm{a}$ & $1,76 \pm 1,09 \mathrm{~b}$ & 0,0324 \\
Neutrófilos $(\%)$ & $46,26 \pm 3,29 \mathrm{a}$ & $53,59 \pm 5,53 \mathrm{~b}$ & 0,0317 \\
Linfócitos $(\%)$ & $91,80 \pm 4,91 \mathrm{a}$ & $83,60 \pm 5,59 \mathrm{~b}$ & 0,0392 \\
Eosinófilos $(\%)$ & $1,56 \pm 0,14 \mathrm{a}$ & $1,13 \pm 0,18 \mathrm{~b}$ & 0,0017 \\
Monócitos $(\%)$ & $0,66 \pm 0,10 \mathrm{a}$ & $0,46 \pm 0,16 \mathrm{a}$ & 0,4206 \\
Basófilos $(\%)$ & $0,39 \pm 0,19 \mathrm{a}$ & $0,33 \pm 0,21 \mathrm{a}$ & 0,4397 \\
\hline
\end{tabular}

${ }^{1}$ Médias seguidas pela mesma letra não diferem significativamente entre si pelo teste de Wilcoxon-Mann-Whitney $(\mathrm{P}<0,05)$.

Tabela 5. ${ }^{1}$ Médias de hematócrito, contagem total e diferencial das células sanguíneas das ratas com 16 dias de gestação

\begin{tabular}{lccc}
\hline & I & II & P \\
\hline Ht $(\%)$ & $41,66 \pm 1,51 \mathrm{a}$ & $42,40 \pm 2,30 \mathrm{a}$ & 0,6905 \\
$\mathrm{CTH}\left(10^{6} / \mu \mathrm{L}\right)$ & $4,77 \pm 0,70 \mathrm{a}$ & $3,64 \pm 1,00 \mathrm{~b}$ & 0,0097 \\
$\mathrm{CTL}\left(10^{3} / \mu \mathrm{L}\right)$ & $2,50 \pm 1,46 \mathrm{a}$ & $1,74 \pm 1,20 \mathrm{~b}$ & 0,0059 \\
Neutrófilos $(\%)$ & $47,00 \pm 2,82 \mathrm{a}$ & $55,25 \pm 2,87 \mathrm{~b}$ & 0,0286 \\
Linfócitos $(\%)$ & $93,60 \pm 4,39 \mathrm{a}$ & $83,80 \pm 4,81 \mathrm{~b}$ & 0,0195 \\
Eosinófilos $(\%)$ & $1,53 \pm 0,17 \mathrm{a}$ & $1,06 \pm 0,15 \mathrm{~b}$ & 0,0159 \\
Monócitos $(\%)$ & $0,63 \pm 0,14 \mathrm{a}$ & $0,60 \pm 0,19 \mathrm{a}$ & 0,3053 \\
Basófilos $(\%)$ & $0,41 \pm 0,24 \mathrm{a}$ & $0,32 \pm 0,23 \mathrm{a}$ & 0,4640 \\
\hline
\end{tabular}

${ }^{\mathrm{T}}$ Médias seguidas pela mesma letra não diferem significativamente entre si pelo teste de Wilcoxon-Mann-Whitney $(\mathrm{P}<0,05)$.

Tabela 6. ${ }^{1}$ Médias de hematócrito, contagem total e diferencial das células sanguíneas das ratas com 18 dias de gestação

\begin{tabular}{lccc}
\hline & I & II & P \\
\hline Ht $(\%)$ & $43,80 \pm 2,65 \mathrm{a}$ & $39,00 \pm 1,58 \mathrm{a}$ & 0,2222 \\
CTH $\left(10^{6} / \mu \mathrm{L}\right)$ & $4,85 \pm 1,51 \mathrm{a}$ & $3,58 \pm 1,17 \mathrm{~b}$ & 0,0118 \\
CTL $\left(10^{3} / \mu \mathrm{L}\right)$ & $2,56 \pm 1,21 \mathrm{a}$ & $1,70 \pm 0,97 \mathrm{~b}$ & 0,0112 \\
Neutrófilos $(\%)$ & $45,75 \pm 3,50 \mathrm{a}$ & $54,00 \pm 5,41 \mathrm{~b}$ & 0,0430 \\
Linfócitos $(\%)$ & $90,75 \pm 4,85 \mathrm{a}$ & $81,00 \pm 6,37 \mathrm{~b}$ & 0,0186 \\
Eosinófilos $(\%)$ & $1,50 \pm 0,18 \mathrm{a}$ & $1,00 \pm 0,27 \mathrm{~b}$ & 0,0240 \\
Monócitos $(\%)$ & $0,58 \pm 0,24 \mathrm{a}$ & $0,41 \pm 0,15 \mathrm{a}$ & 0,2364 \\
Basófilos $(\%)$ & $0,49 \pm 0,21 \mathrm{a}$ & $0,42 \pm 0,20 \mathrm{a}$ & 0,9999 \\
\hline
\end{tabular}

${ }^{\mathrm{T}}$ Médias seguidas pela mesma letra não diferem significativamente entre si pelo teste de Wilcoxon-Mann-Whitney $(\mathrm{P}<0,05)$.

Tabela 7. ${ }^{1}$ Médias de hematócrito, contagem total e diferencial das células sanguíneas das ratas com 20 dias de gestação

\begin{tabular}{lccc}
\hline & I & II & P \\
\hline Ht $(\%)$ & $42,80 \pm 4,32 \mathrm{a}$ & $36,60 \pm 3,05 \mathrm{~b}$ & 0,0159 \\
$\mathrm{CTH}\left(10^{6} / \mu \mathrm{L}\right)$ & $4,88 \pm 1,02 \mathrm{a}$ & $3,51 \pm 1,84 \mathrm{~b}$ & 0,0109 \\
$\mathrm{CTL}\left(10^{3} / \mu \mathrm{L}\right)$ & $2,61 \pm 1,41 \mathrm{a}$ & $1,68 \pm 1,15 \mathrm{~b}$ & 0,0204 \\
Neutrófilos $(\%)$ & $49,75 \pm 4,64 \mathrm{a}$ & $58,25 \pm 1,70 \mathrm{~b}$ & 0,0139 \\
Linfócitos $(\%)$ & $88,25 \pm 1,70 \mathrm{a}$ & $80,75 \pm 2,63 \mathrm{~b}$ & 0,0031 \\
Eosinófilos $(\%)$ & $1,46 \pm 0,17 \mathrm{a}$ & $1,00 \pm 0,24 \mathrm{~b}$ & 0,0079 \\
Monócitos $(\%)$ & $0,66 \pm 0,47 \mathrm{a}$ & $0,50 \pm 0,0,42 \mathrm{a}$ & 0,6230 \\
Basófilos $(\%)$ & $0,50 \pm 0,42 \mathrm{a}$ & $0,41 \pm 0,31 \mathrm{a}$ & 0,7601 \\
\hline
\end{tabular}

${ }^{1}$ Médias seguidas pela mesma letra não diferem significativamente entre si pelo teste de Wilcoxon-Mann-Whitney $(\mathrm{P}<0,05)$. 
Tabela 8. ${ }^{1}$ Médias do diâmetro $(\mu \mathrm{m})$ das hemácias das ratas com 14 e 21 dias de gestação

\begin{tabular}{cccccc}
\hline Grupos & I & II & III & IV & P \\
\hline 14 dias & $6,58 \pm 0,26 \mathrm{a}$ & $5,77 \pm 0,05 \mathrm{~b}$ & $6,82 \pm 0,12 \mathrm{a}$ & $6,75 \pm 0,07 \mathrm{a}$ & 0,0082 \\
21 dias & $6,73 \pm 0,05 \mathrm{a}$ & $5,49 \pm 0,14 \mathrm{~b}$ & $6,70 \pm 0,22 \mathrm{a}$ & $6,81 \pm 0,18 \mathrm{a}$ & 0,0085
\end{tabular}

${ }^{\mathrm{T}}$ Médias seguidas pela mesma letra não diferem significativamente entre si pelo teste de Wilcoxon-Mann-Whitney $(\mathrm{P}<0,05)$.

Os níveis séricos de glicose nas matrizes no $7^{\circ} \mathrm{e}$ $14^{\circ}$ dia de prenhez não apresentaram variação significativa, porém houve diminuição no grupo II em relação aos outros grupos experimentais no $21^{\circ}$ dia de gestação. A dosagem dos níveis séricos de progesterona revelou uma diminuição significativa desse hormônio aos 14 e 21 dias de prenhez nas ratas do grupo tratado com dexametasona (Tab. 9). Estatisticamente não houve influência dos tratamentos sobre os parâmetros morfométricos no fígado, rins e adrenais (Tab. 10). Também não foram evidenciadas alterações histoquímicas nesses órgãos (Fig. 1 e 2).

Tabela 9. ${ }^{1}$ Médias dos níveis séricos de carboidratos totais $(\mathrm{mg} / \mathrm{dL})$ e progesterona $(\mathrm{ng} / \mathrm{mL})$ nas ratas dos grupos experimentais

\begin{tabular}{|c|c|c|c|c|c|}
\hline \multicolumn{6}{|c|}{ Grupos } \\
\hline & GI & GII & GIII & GIV & $\mathrm{P}$ \\
\hline \multicolumn{6}{|c|}{ Carboidratos } \\
\hline $7^{\mathrm{o}}$ & $452,44 \pm 50,51 \mathrm{a}$ & $427,11 \pm 82,27 \mathrm{a}$ & $498,83 \pm 29,57 a$ & $398,60 \pm 67,35 a$ & 0,0845 \\
\hline $14^{\mathrm{o}}$ & $314,61 \pm 70,06 a$ & $359,15 \pm 81,03 \mathrm{a}$ & $427,95 \pm 47,95 \mathrm{a}$ & $318,03 \pm 80,73 a$ & 0,0797 \\
\hline $21^{\mathrm{o}}$ & $476,36 \pm 47,20 \mathrm{a}$ & $227,77 \pm 53,69 b$ & $396,40 \pm 53,53 a$ & $419,76 \pm 49,29 a$ & 0,0029 \\
\hline \multicolumn{6}{|c|}{ Progesterona } \\
\hline $7^{\mathrm{o}}$ & $662,93 \pm 9,97 a$ & $659,36 \pm 5,72 a$ & $666,19 \pm 6,56 a$ & $667,08 \pm 4,70 a$ & 0,8621 \\
\hline $14^{\mathrm{o}}$ & $686,38 \pm 8,45 a$ & $621,95 \pm 4,15 b$ & $694,99 \pm 4,49 a$ & $690,84 \pm 3,86 a$ & 0,0078 \\
\hline $21^{\mathrm{o}}$ & $697,66 \pm 4,20 \mathrm{a}$ & $632,94 \pm 7,80 b$ & $699,15 \pm 5,35 a$ & $695,29 \pm 2,71 \mathrm{a}$ & 0,0166 \\
\hline
\end{tabular}

${ }^{\mathrm{T}}$ Médias seguidas pela mesma letra não diferem significativamente entre si pelo teste de Wilcoxon-Mann-Whitney $(\mathrm{P}<0,05)$.

Tabela 10. ${ }^{1}$ Médias do percentual do parênquima lobular (PL), não lobular do fígado (PNL), área da cortical (AC) e Medular (AM) da adrenal, diâmetro (DG) e volume do glomérulo (VG), diâmetro (DCB) e volume (VCB) da cápsula de Bowman dos rins nos grupos experimentais

\begin{tabular}{|c|c|c|c|c|c|}
\hline \multicolumn{6}{|c|}{ Grupos } \\
\hline Órgãos & GI & GII & GIII & GIV & $\mathrm{P}$ \\
\hline \multicolumn{6}{|l|}{ Fígado } \\
\hline PL (\%) & $91,61 \pm 5,75 \mathrm{a}$ & $95,90 \pm 1,68 \mathrm{a}$ & $96,27 \pm 1,83 a$ & $93,52 \pm 3,77 a$ & 0,2125 \\
\hline $\begin{array}{l}\text { PNL } \\
(\%)\end{array}$ & $8,38 \pm 3,25 a$ & $4,10 \pm 1,75 \mathrm{a}$ & $3,72 \pm 1,28 \mathrm{a}$ & $6,47 \pm 3,68 \mathrm{a}$ & 0,1918 \\
\hline \multicolumn{6}{|l|}{ Adrenal } \\
\hline $\begin{array}{c}\mathrm{AC} \\
\left(\mu \mathrm{m}^{2}\right)\end{array}$ & $4030,97 \pm 56,64 a$ & $3956,85 \pm 166,88 \mathrm{a}$ & $4077,25 \pm 188,77 \mathrm{a}$ & $3988,89 \pm 153,35 a$ & 0,9192 \\
\hline $\begin{array}{c}\mathrm{AM} \\
\left(\mu \mathrm{m}^{2}\right)\end{array}$ & $1259,70 \pm 67,92 \mathrm{a}$ & $1270,44 \pm 38,59 a$ & $1257,95 \pm 50,41 \mathrm{a}$ & $1260,91 \pm 50,64 \mathrm{a}$ & 0,2564 \\
\hline \multicolumn{6}{|l|}{ Rim } \\
\hline $\begin{array}{l}\mathrm{DG} \\
(\mu \mathrm{m})\end{array}$ & $128,58 \pm 14,53 \mathrm{a}$ & $134,66 \pm 12,86 \mathrm{a}$ & $125,54 \pm 18,42 \mathrm{a}$ & $137,85 \pm 12,52$ & 0,0687 \\
\hline $\begin{array}{c}\mathrm{VG} \\
\left(\mu \mathrm{m}^{3}\right)\end{array}$ & $1271342,81 \pm 280111 \mathrm{a}$ & $11666049,00 \pm 181883 a$ & $1088850,93 \pm 236535 \mathrm{a}$ & $1233284,67 \pm 305569 a$ & 0,6435 \\
\hline $\begin{array}{l}\text { DCB } \\
(\mu \mathrm{m})\end{array}$ & $146,40 \pm 13,78 \mathrm{a}$ & $138,63 \pm 11,59 a$ & $144,93 \pm 12,54 \mathrm{a}$ & $147,33 \pm 15,66 a$ & 0,2649 \\
\hline $\begin{array}{l}\mathrm{VCB} \\
\left(\mu \mathrm{m}^{3}\right)\end{array}$ & $1336238,24 \pm 218257 \mathrm{a}$ & $1513826,85 \pm 239069 \mathrm{a}$ & $1313852,17 \pm 265163 \mathrm{a}$ & $1375855,34 \pm 252962 a$ & 0,1618 \\
\hline
\end{tabular}



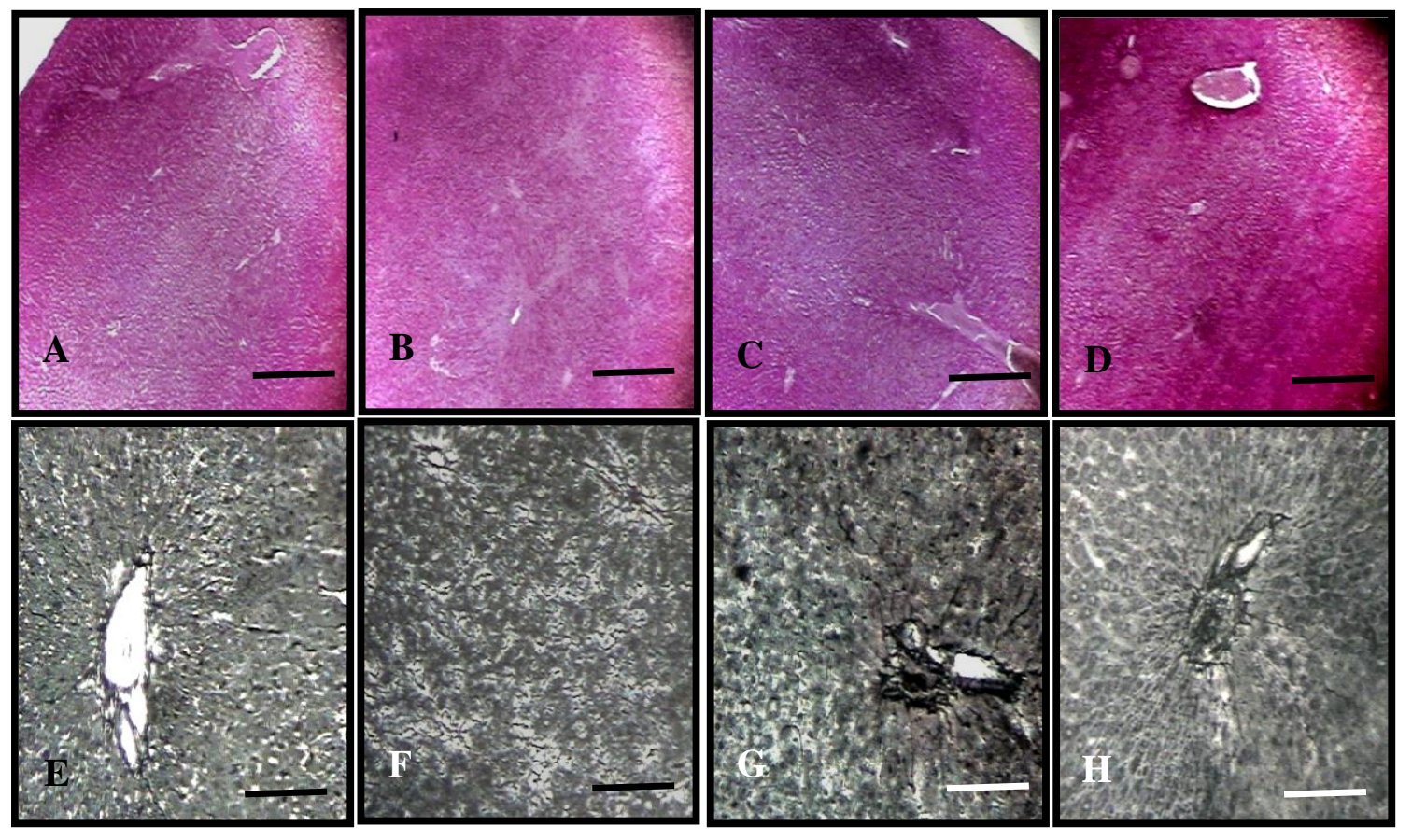

Figura 1. Micrografia do fígado das ratas dos grupos experimentais coradas com P.A.S.: A - Grupo I, B Grupo II, C - Grupo III, D - Grupo IV (Barras = 200 $\mu \mathrm{m})$; e pela impregnação argêntica: E - Grupo I, F Grupo II, G - Grupo III, H - Grupo IV (Barras = 100 $\mu \mathrm{m}$ ).
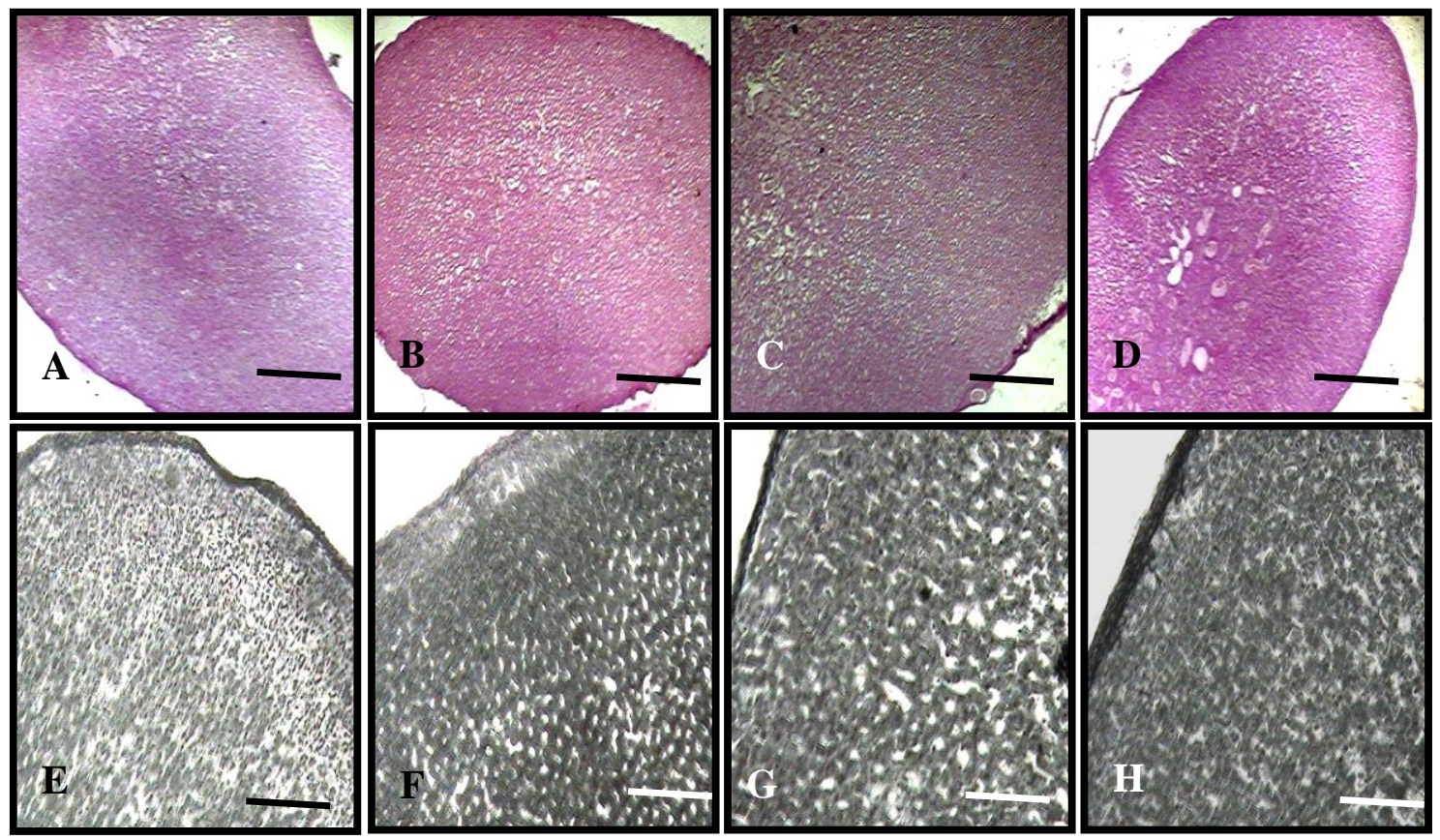

Figura 2. Micrografia da Adrenal das ratas dos grupos experimentais coradas com P.A.S.: A - Grupo I, B

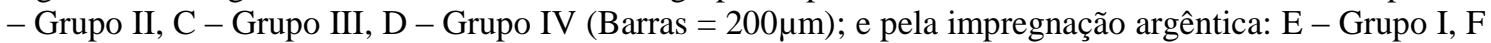

- Grupo II, G - Grupo III, H - Grupo IV (Barras = 100 $\mu \mathrm{m})$. 


\section{DISCUSSÃO}

No presente trabalho, no $14^{\circ}$ e no $21^{\circ}$ dia de gestação, observou-se redução significativa no número de hemácias nas ratas do grupo II, o que pode estar associado à redução da eritropoese. Além disso, verificou-se ainda uma anisocitose do tipo micrócito nas hemácias, sugerindo um quadro de anemia, ao passo que, nos demais grupos, essa característica manteve-se normal, inclusive no grupo IV. Segundo Liu et al. (2013), a dexametasona reduz a síntese de eritropoetina em situações de hipóxia; isso poderia afetar a eritropoese, reduzido o número de hemácias.

Experimentos com camundongos fêmeas (Swiss Webster), administrando doses imunossupressoras $(5 \mathrm{mg} / \mathrm{kg})$ e anti-inflamatórias $(1,5 \mathrm{mg} / \mathrm{kg})$ de dexametasona i.p., durante 12 dias, promoveram redução mais acentuada no número total de leucócitos no grupo que recebeu dose imunossupressora (Cupolilo et al., 2007). Em nossos estudos, encontramos resultado semelhante aos 14 dias de gestação, além de neutrofilia, linfopenia e eosinopenia nas ratas do grupo II em relação aos outros grupos experimentais. No terço final $\left(21^{\circ}\right.$ dia $)$ da gestação, esses resultados foram mantidos. Isso revela que a administração contínua a partir do $10^{\circ}$ dia até o fim da gestação, mesmo com a redução progressiva da dose, possui efeitos imunossupressores.

Foi observado um aumento no percentual de hematócrito nas ratas do grupo II no $14^{\circ}$ dia de prenhez, e redução ao $21^{\circ}$ dia, associados à diminuição do diâmetro das hemácias nesses períodos. Sabe-se que os glicocorticoides exercem efeitos sobre o mecanismo de homeostase dos fluidos corporais, provocando alterações, como redução da volemia e aumento da osmolalidade do sangue (Ruginsk et al., 2007). Postula-se que a administração aguda ou crônica de glicocorticoides pode levar, respectivamente, ao aumento ou redução da diurese e natriurese (Liu et al., 2007), além de promover alterações no citoesqueleto das células (Aru et al., 2013). Assim, a variação no percentual de hematócrito pode estar relacionada a uma disfunção renal, levando no início a um quadro de desidratação, causa mais comum de aumento do hematócrito (Ribeiro Filho et al., 2011), e, no período final, a um possível quadro de anemia (Bejarano et al., 2003). Isso sugere que, dependendo da dose, os glicocorticoides podem interferir na homeostase de líquidos extracelulares nas fases finais da prenhez, além de afetar significativamente a estrutura celular das hemácias.

A melatonina associada à dexametasona exerceu um efeito protetor em todos os parâmetros sanguíneos analisados. Estudos realizados por Lissoni et al. (2003) em pacientes com câncer de pulmão, submetidos a quimioterápicos contendo cisplatina, conhecida por induzir a anemia, observaram que os níveis de hemoglobina eram maiores quando os pacientes eram tratados concomitantemente com melatonina. Segundo Srinivasan et al. (2005), a melatonina é benéfica tanto à resposta imune celular quanto à humoral, estimulando a produção de citocinas e células progenitoras dos granulócitos, macrófagos, células $\mathrm{NK}$ e algumas células $\mathrm{T}$ auxiliares (CD4+). Isso explica o efeito imunoprotetor exercido pela melatonina quando administrada nas ratas do grupo IV.

Santos et al. (2007) administraram dexametasona $(1 \mathrm{mg} / \mathrm{kg})$, em diferentes períodos $(24,72 \mathrm{e}$ $120 \mathrm{~h}$ ), em ratos adultos, e observaram que os efeitos sobre o metabolismo são dependentes da dose e do tempo de administração, pois ocorreu um aumento dos níveis de insulina plasmática após 24 horas, juntamente com pequena elevação das concentrações de glicose sanguínea, aumentando consideravelmente nos animais tratados por 120 horas, estabelecendo um quadro de resistência à insulina. Em nosso estudo, porém, os níveis plasmáticos de glicose nas ratas tratadas com dexametasona, no $14^{\circ}$ dia de prenhez, não apresentaram variação significativa; e, no $21^{\circ}$ dia de prenhez, ocorreu uma diminuição desses níveis. Uma vez que foi realizada a redução progressiva diária da dose administrada, pode ter ocorrido uma resposta adaptativa das células $\beta$ pancreáticas; pois, durante a gestação, ocorrem adaptações no metabolismo de carboidratos, culminando com um progressivo estado de resistência à insulina, que impedem a metabolização da glicose materna, elevando os seus níveis no início da gestação (Ericsson et al., 2007). No entanto, a administração de dexametasona durante o terço final $\left(14^{\circ}\right.$ ao $19^{\circ}$ dia $)$ estimula a secreção de insulina, suprimindo a produção endógena de glicose, levando à redução dos seus níveis séricos (Holness e Sudgen, 2001). 
Os níveis séricos de progesterona mostraram-se reduzidos aos 14 e 21 dias de prenhez apenas nas ratas do grupo II. De acordo com Wang et al. (1993), a aplicação de dexametasona até 12 dias aumenta significativamente o nível de progesterona, em até 16 dias os níveis séricos desse hormônio são reduzidos em cerca de $50 \%$ e completamente deprimidos após 19 dias de tratamento. No tratamento dexametasona associado com melatonina (grupo IV), verificouse um efeito protetor da melatonina, mantendo normais os níveis séricos de progesterona. Segundo Tamura et al. (2008) e Dair et al. (2008), a melatonina, dentre outras funções, estimula a secreção da progesterona, o que reduz a contratilidade uterina e evita a rejeição imunológica do trofoblasto, eventos necessários para uma gravidez bem-sucedida.

A não alteração dos parâmetros morfométricos e histoquímicos no fígado, rins e adrenais pode estar relacionada ao tempo de atividade da dexametasona; pois, segundo Pauli et al. (2005), esse glicocorticoide apresenta uma meia vida que varia entre 36 e 54 horas. Outro fator que poderia explicar esse resultado estaria relacionado ao desmame efetuado nas ratas tratadas com dexametasona.

\section{CONCLUSÃO}

A dexametasona na dosagem de $0,8 \mathrm{mg} / \mathrm{kg}$, administrada a partir do terço médio da gestação, produz alterações hematológicas, bioquímicas e hormonais em ratas, sendo esses efeitos prevenidos pela melatonina, entretanto essa dosagem não afeta o fígado, rins e adrenais quanto aos parâmetros morfométricos e histoquímicos.

\section{REFERÊNCIAS}

AKAOKA, K.; WHITE, R.H.; RAAFAT, F. Human glomerular growth during childhood: a morphometric study. J. Pathol., v.173, p.261-268, 1994.

AOYAMA, H.; MORI, W.; MORI, N. Antiglucocorticoid effects of melatonin in young rats. Acta Pathol. Jpn., v.36, p.423-428, 1986.

ARU, M.; ALEV, K.; GAPEYEVA, H. et al. Glucocorticoid-induced alterations in titin, nebulin, myosin heavy chain isoform content and viscoelastic properties of rat skeletal muscle. Adv. Biol. Chem., v.3, p.70-75, 2013.
BAVARESCO, L.; BERNARDI, A.; BATTASTINI, A.M.O. Glicocorticoides: Usos clássicos e emprego no tratamento do câncer. Infarma., v.17, p.58-60, 2005.

BEJARANO, I.F.; DIPIERRI, J.E.; ALFARO, E.L. $e t$ al. Valores del hematocrito y prevalencia de anemia en escolares jujeños. Medicina, v.63, p.288-292, 2003.

BRUDER, E.D.; PING, C.L.; RAFF, H. Metabolic consequences of hypoxia from birth and dexamethasone treatment in the neonatal rat: comprehensive hepatic lipid and fatty acid profiling. Endocrinology., v.145, p.5364-5372, 2006.

CUPOLILO, S.M.N.; GOLLNER, A.M.; SOUZA, R.L.P. et al. Investigação de efeitos lesivos de doses antiinflamatórias e imunossupressoras de dexametasona sobre a mucosa gástrica em camundongos. Hu. rev., v.33, p.17-22, 2007.

DAIR, E.L.; SIMÕES, R.S.; SIMÕES, M.J. et al. Effects of melatonin on the endometrial morphology and embryo implantation in rats. Fertil. Steril., v.89, p.1299-1305, 2008 .

DHABHAR, F.S.; MILLER, A.H.; MCEWEN, B.S.; SPENCER, R.L. Stress-induced changes in blood leukocyte distribution. Role of adrenal steroid hormones. J. Immunol., v.157, p.1638-1644, 1996.

ENGELMAN, M.F.B.; NETO, J.G.; ANDRADE, C.H.V. et al. Estudo morfométrico do fígado de ratos submetidos a doses supra-fisiológica de tiroxina. Arq. Bras. Endocrinol. Metab., v.45, p.173-179, 2001.

ERICSSON, A.; SALJO, K.; SJOSTRAND, E. et al. Brief hyperglycaemia in the early pregnant rat increases fetal weight at term by stimulating placental growth and affecting placental nutrient transport. $J$. Physiol., v.581, p.1323-1332, 2007.

HOLNESS, M.J.; SUGDEN, M.C. Dexamethasone during Late Gestation Exacerbates Peripheral Insulin Resistance and Selectively Targets Glucose-Sensitive Functions in $\beta$ Cell and Liver. Endocrinol., v.142, p.3742-3748, 2001.

LAURENTINI, A.; EDWARDS, C.A. A microtiter modification of the anthrone-sulfuric acid colorimetric assy for glucose-based carbohydrates. Anal. Biochem., v.315, p.413-415, 2003.

LEE, J.Y.; PARK, S.J.; KIM, S.H. Prenatal administration of dexamethasone during early pregnancy negatively affects placental development and function in mice. J. Anim. Sci., v.90, p.4846-4856, 2012.

LISSONI, P.; MALUGANI, F.; BUKOVEC, R. et al. Reduction of cisplatin-induced anemia by the pineal indole 5-methoxytryptamine in metastatic lung cancer patients. Neuro. Endocrinol. Lett., v.24, p.83-85, 2003. 
LIU, C.; LIU, G.; ZHOU, C. et al. Potent diuretic effects of prednisone in heart failure patients with refractory diuretic resistance. Can. J. Cardiol., v.23, p.865-868, 2007.

LIU, C.; CROFT, Q.P.; KALIDHAR, S. et al. Dexamethasone mimics aspects of physiological acclimatization to 8 hours of hypoxia but suppresses plasma erythropoietin. J. Appl. Physiol., v.114, p.948956, 2013.

LOCATELLI, A.; GHIDINI, A.; INCERTI, M. et al. Gestational age at glucocorticoids administration after premature rupture of membranes and cerebral white matter damage. J. Matern. Fetal Neonatal. Med., v.23, p.511-515, 2010

PAULI, J.R.; LEME, J.; CRESPILHO, D. et al Influência do treinamento físico sobre parâmetros do eixo hipotálamo-pituitária-adrenal de ratos administrados com dexametasona. Rev. Port. Cienc. Desp., v.5, p.143-152, 2005.

RIBEIRO FILHO, J.D.; GIMENES, A.M.; FONSECA, E.F. et al. Enteral fluid therapy in cattle: evaluation of isotonic electrolyte solutions administered via nasogastric tube on continuous flow. Cienc. Rural., v.41, p.285-290, 2011.

REMMELTS, H.H.F.; MEIJVIS, S.C.A.; BIESMA, D.H. et al. Dexamethasone downregulates the systemic cytokine response in patients with community-acquired pneumonia. Clin. Vaccine Immunol., v.19, p.1532-1538, 2012.

RHODUS, N.L.; CHENG, B.; BOWLES, W. et al. Proinflammatory cytokine levels in saliva before and after treatment of (erosive) oral lichen planus with dexamethasone. Oral DIs., v.12, p.112-116, 2006.
RUGINSK, S.G.; OLIVEIRA, F.R.; MARGATHO, L.O. et al. Glucocorticoid modulation of neuronal activity and hormone secretion induced by blood volume expansion. Exp. Neurol., v.206, p.192-200, 2007.

SANTOS, C.L.; RAFACHO, A.; BOSQUEIRO, J.R. Efeitos da administração de dexametasona in vivo sobre glicemia, insulinemia e substratos circulantes são dependentes do tempo de tratamento. Biosci. J., v.23, p.101-110, 2007.

SRINIVASAN, V.; MAESTRONI, G.F.; CARDINALI, D.P. et al. Melatonin, immune function and aging. Immun. Ageing., v.29, p.17, 2005.

SUBRAMANIAN, P.; MIRUNALINI, S.; DAKSHAYANI, K.B. et al. Prevention by melatonin of hepatocarcinogenesis in rats injected with $\mathrm{N}$ nitrosodiethylamine. J. Pineal Res., v.43, p.305-312, 2007.

TAMURA, H.; NAKAMURA, Y.; TERRON, M.P. et al. Melatonin and pregnancy in the human. Reproduc. Toxicol., v.25, p.291-303, 2008.

VILAÇA-JÚNIOR, P.E.A.; SOARES, A.F.; WANDERLEY-TEIXEIRA, V. et al. Efeito da administração pré-natal da dexametasona em ratas sobre os perfis glicídicos e hematológicos materno e da prole. Arq. Bras. Med. Vet. Zootec., v.64, p.606614, 2012

WANG, F.; RILEY, J.C.M.; BEHRMAN, H.R. Immunosuppressive levels of glucocorticoid block extrauterine luteolysins in the rat. Biol. Reprod., v.49, p.66-73, 1993. 